Published as Parallel Processing Letters (PPL) 22 (2012) 1240009 (12 pages)

DOI: 10.1142/S0129626412400099 @CWorld Scientific Publishing Company

\title{
MATHEMATICAL FACTS IN A PHYSICALIST ONTOLOGY
}

\author{
LÁSZLÓ E. SZABÓ \\ Department of Logic, Institute of Philosophy \\ Eötvös University, Budapest \\ Múzeum krt. 4/i Budapest, H-1088 Hungary \\ http://phil.elte.hu/leszabo
}

\begin{abstract}
If physicalism is true, everything is physical. In other words, everything supervenes on, or is necessitated by, the physical. Accordingly, if there are logical/mathematical facts, they must be necessitated by the physical facts of the world. The aim of this paper is to clarify what logical/mathematical facts actually are and how these facts can be accommodated in a purely physical world.
\end{abstract}

\section{Introduction}

If physicalism is true, everything is physical. In other words, everything supervenes on, or is necessitated by, the physical. In a coherent physicalist ontology there is no room for abstract and mental entities with which logic and mathematics are routinely associated. Accordingly, if there are logical/mathematical facts, they must be understood in terms of the physical reality; they must be necessitated by the physical facts of the world. So, in a physicalist ontology, the following two questions must be answered: 1) What does a logical/mathematical fact consist in? In other words: What is it that must be necessitated by the physical facts of the world? 2) How does this necessitation come about? The aim of a physicalist philosophy of mathematics (first outlined in [1]) is to clarify these questions and to explain how logic and mathematics can be accommodated in a purely physical ontology. In this paper, I shall articulate the basic 
ideas, and provide a few more arguments for the physicalist interpretation of formal systems.

To avoid any misunderstanding, it is worthwhile to make a distinction: what I call physicalist philosophy of mathematics has nothing to do with immanent/physical realism or naturalism (as in $[2,3,4,5]$ ). According to the latter the mathematical concepts and propositions reflect some fundamental features of the physical world. In contrast, the proposed physicalist interpretation of formal logic and mathematics will be based on the strongest formalist approach to mathematics; according to which mathematical objects carry no meanings whatsoever. "The formulas are not about anything; they are just strings of symbols" ([6], p. 319); or, as Hilbert allegedly expressed this idea in a famous aphorism about Euclidean geometry: "One must be able to say at all timesinstead of points, straight lines, and planes - tables, chairs, and beer mugs" ([7], p. 81).

\section{Truth in a physical theory}

To avoid any misunderstanding later in the paper, let us first clarify the essential difference between mathematical "truth" and a semantic truth in a physical theory describing something in the world. A physical theory $(L, S)$ is a formal system $L$ with a partial semantics $S$, where $S$ is pointing to the (empirical) world. In general, $L$ is a (first-order) formal language with some logical axioms and the derivation rules (usually the first-order predicate calculus with identity), the axioms of certain mathematical theories, and, of course, some physical axioms.

Consider a sentence $A$ in physical theory $(L, S)$. One has to distinguish the following two cases:

To be a theorem: $L \vdash A$ (a fact of the formal system $L$ ).

To be true: $\quad$ According to the semantics $S, A$ refers to a state of affairs in the physical world, which is in fact the case (a fact of the physical system described by the theory).

For example, 'The electric field strength of a point charge is $\frac{k Q}{r^{2}}$ ' is a theorem of Maxwell's electrodynamics - one can derive the corresponding formal expression from the Maxwell equations. (This is a fact of the formal system L.) On the other hand, according to the semantics relating the symbols of the Maxwell theory to the empirical terms, this sentence corresponds to an empirical fact (about the point charges).

From an epistemological point of view, to be a theorem and to be true are different and independent concepts, in the sense that one does not automatically imply the other. Of course, one of the aims of a physical theory is to keep the two things in synchrony throughout the region of validity of the theory in question. However, assume that $\Gamma$ is a set of true sentences in $L$, i.e., each sentence in $\Gamma$ refers to an empirical fact, and also assume that $\Gamma \vdash A$ in $L$. It does not 
automatically follow that $A$ is true. Whether $A$ is true is again an empirical question. If so, then it is a new empirically obtained information about the world, confirming the validity of the whole physical theory $(L, S)$.

But if it turns out that $A$ is not true, then this information falsifies the physical theory, as a whole. That is to say, one has to think about revising one of the constituents of $(L, S)$, the physical axioms, the semantics $S$, the mathematical axioms, or the axioms of logic or the derivation rules of $L-$ probably in this order.

\section{What are logical/mathematical facts?}

Having clarified the difference between being true and being derived, it is worthwhile briefly reviewing the main argument for the formalist thesis that mathematical propositions have no meanings - even if, as platonists and intuitionists assume, these meanings would refer not to the physical but to the platonic or to the mental realms. The argument is, in some weak sense, based on the truthcondition theory of meaning. If we accept that "a meaning for a sentence is something that determines the conditions under which the sentence is true or false" ([8], p. 173), and the physical realist/platonist/intuitionist understanding of mathematics is correct, then the truth-condition of a mathematical proposition must be found in the physical/platonic/mental world. In that case, in the verification of a mathematical statement, there must occur some reference both to the state of affairs in the physical/platonic/mental world and to the means by which we have epistemic access to these worlds. But there is no trace of this in the practice of mathematics.

For example, consider a very simple mathematical theory: the theory of groups (Fig. 1). What will the mathematician answer to the following questions: "Why is $p(e, p(e, e))=e$ true?"; "How do we know that $p(e, p(e, e))=e$ is true?"; "How can we verify that $p(e, p(e, e))=e$ is true?"? In answering these question, the mathematician never even mentions how the things are in the physical/platonic/mental world and never even mentions the epistemic means by which we have access to these realms. For the mathematician's final argument is that formula $p(e, p(e, e))=e$ has a proof in group theory; and (s)he will show us an evidence like in Fig. 2. In Dummett's words:

Like the empiricist view, the platonist one fails to do justice to the role of proof in mathematics. For, presumably, the supra-sensible realm is as much God's creature as is the sensible one; if so, conditions in it must be as contingent as in the latter. [...] [W] do not seek, in order to refute or confirm a [mathematical] hypothesis, a means of refining our intuitive faculties, as astronomers seek to improve their instruments. Rather, if we suppose the hypothesis true, we seek for a proof of it, and it remains a mere hypothesis, whose assertion would therefore be unwarranted, until we find one. ([9], p. 13) 


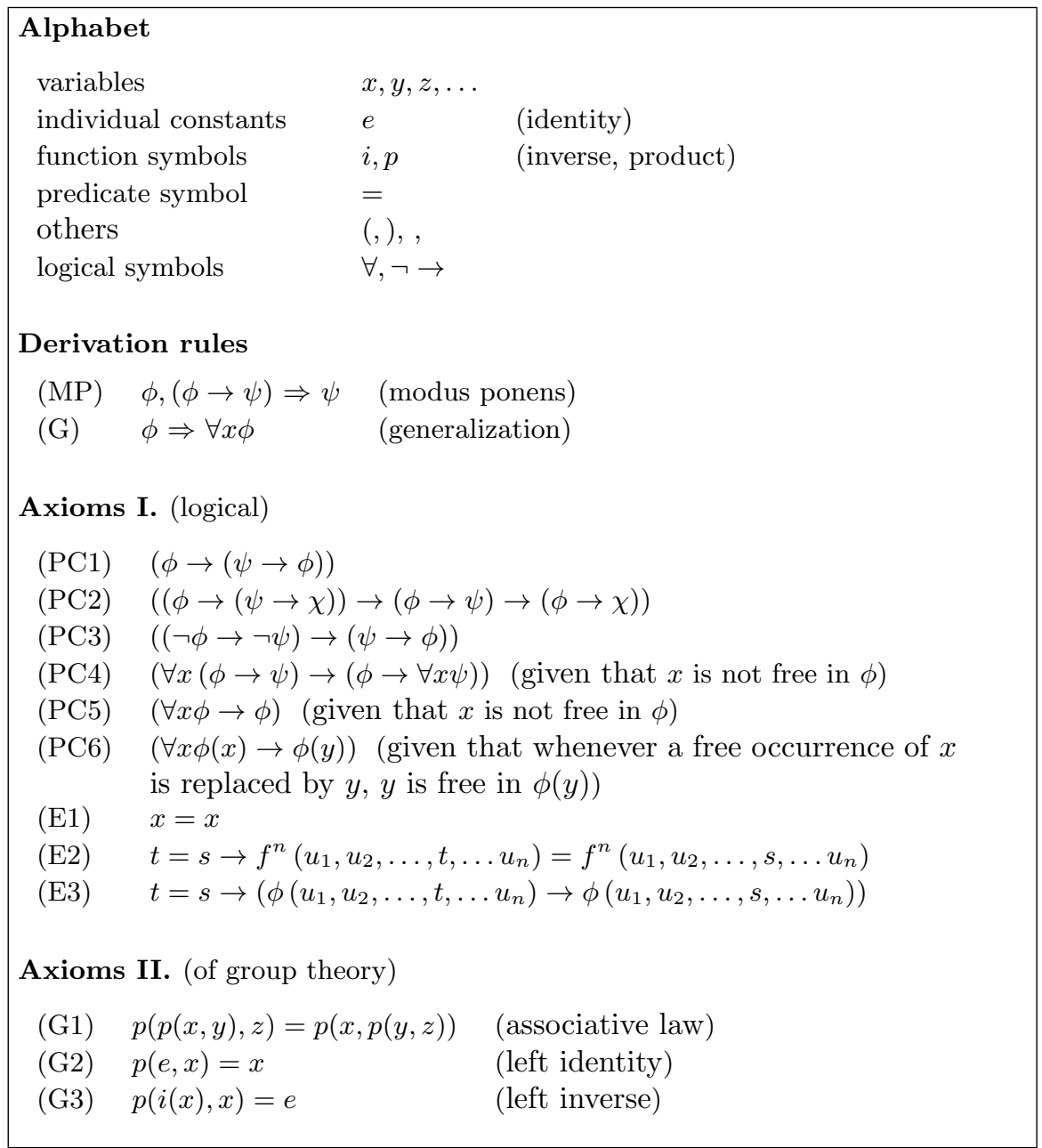

Figure 1: Group theory

\begin{tabular}{|lll|}
\hline$(1)$ & $p(e, x)=x$ & $(\mathrm{G} 2)$ \\
$(2)$ & $(\forall x)(p(e, x)=x)$ & $(\mathrm{G})$ \\
$(3)$ & $(\forall x)(p(e, x)=x) \rightarrow p(e, e)=e$ & $(\mathrm{PC} 6)$ \\
$(4)$ & $p(e, e)=e$ & $(2),(3),(\mathrm{MP})$ \\
$(5)$ & $(\forall x)(p(e, x)=x) \rightarrow p(e, p(e, e))=p(e, e)$ & $(\mathrm{PC} 6)$ \\
$(6)$ & $p(e, p(e, e))=p(e, e)$ & $(2),(5),(\mathrm{MP})$ \\
$(7)$ & $p(e, e)=e \rightarrow p(e, p(e, e))=p(e, e) \rightarrow p(e, p(e, e))=e$ & $(\mathrm{E} 3)$ \\
$(8)$ & $p(e, p(e, e))=p(e, e) \rightarrow p(e, p(e, e))=e$ & $(4),(7),(\mathrm{MP})$ \\
$(9)$ & $p(e, p(e, e))=e$ & $(6),(8),(\mathrm{MP})$ \\
\hline
\end{tabular}

Figure 2: The proof of $p(e, p(e, e))=e$ 
Therefore, a mathematical proposition (like " $p(e, p(e, e))=e$ ") does not have meaning; it does not refer to anything and cannot be true or false in the ordinary semantical sense (like a sentence of a physical theory). It is actually not a linguistic object, it is just a "brick" in a formal system-consequently, it does not express a fact whatsoever.

It is important to note that one must not confuse such a mathematical "brick" with the meta-mathematical sentence stating the provability of this "brick"; for example, " $p(e, p(e, e))=e$ " with " $\{$ Group $\} \vdash p(e, p(e, e))=e$ ". The latter is a meaningful meta-mathematical sentence, asserting a property of the formal system called group theory, namely that there is a proof of $p(e, p(e, e))=$ $e$ in the system. The validity of this statement can be verified by the close observation of Figure 2. This is what can be considered as a mathematical fact.

\section{Ontology of formal systems}

Now we arrive at the point where the physicalist approach I propose becomes different from the standard formalist philosophy of mathematics. The question we are asking now is: Where are the states of affairs located in the ontological picture of the world that make the meta-mathematical propositions like " $\{$ Group $\} \vdash p(e, p(e, e))=e$ " true or false? My main thesis is that a formal system must be regarded as a physical system which consists of signs and derivational mechanisms embodied in concrete physical objects and concrete physical processes. Therefore, a $\Sigma \vdash \phi$-type meta-mathematical proposition expresses an objective fact of the physical world, namely, a fact of the formal system itself as a particular portion of the physical world.

I will argue for this physicalist account of formal systems in three steps. I shall show that

(I) A formal systems can be represented in a physical system.

(II) We have access to a formal system only in some concrete physical representation.

(III) Actually, there is nothing to be "represented"; there is nothing beyond the flesh and blood physical "representations".

(I) Let me start with a commonly accepted view. Imagine a notebook with an inserted CD (Fig. 3). The CD contains a program completely determining the notebook's behavior: in some order, the computer lists the theorems and the proofs of a formal system. I think, it is commonly accepted to say that in the "computer + CD" system we have "a physical representation of the formal system" in question. In this representation, it is obviously true that the fact of whether or not a given formula $\phi$ is a theorem, that is, whether or not the computer will print out formula $\phi$ to the screen, is a fact of the physical world; namely, it is entirely determined by the physical process going on within 


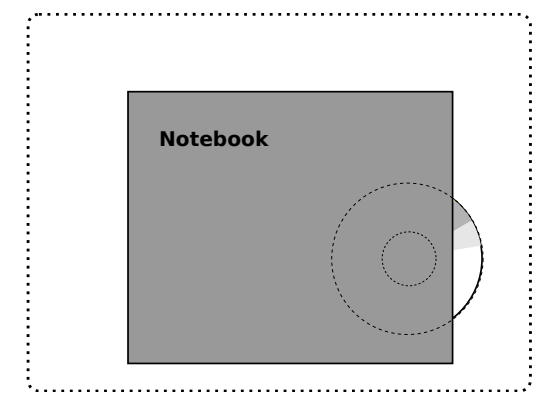

Figure 3: A formal system represented in a notebook with a $C D$. The $C D$ contains a program making the computer to list the theorems of the formal system

the region symbolized by the dotted line (Fig. 3); it is predetermined by the physical laws and the initial state of the computer and the CD - note that a "program" is nothing but a certain physical state of the surface of the CD. Thus, in this physical representation, the statement that $\Sigma \vdash \phi$ is an ordinary scientific statement:

(1) it expresses an objective fact of the physical world;

(2) it can be true before anybody discovers it;

(3) it is a posteriori, and accordingly, not necessary and not certain.

Points (1) and (2) are quite obvious; they are straightforward consequences of the fact that $\Sigma \vdash \phi$ asserts the existence of a physical process inside of the dotted line, irrespectively of whether anybody observes this process or not, and irrespectively of the fact that a computer is an artifact - a constellation in the physical world caused by mankind. Point (2) holds even in a stronger sense. $\Sigma \vdash \phi$ actually asserts the existence of a physical process, given certain initial conditions within the dotted line - to be a 'notebook + CD' in the initial state. The laws of nature probably predetermine whether this process is possible or not, even if nobody has initiated such a process yet. This simply refers to the normal situation in sciences. For example, it was true that the laws of nature admitted a chemical process ending with a plastic, say PVC (polyvinyl chloride), molecule, even before the chemist Eugen Baumann discovered the existence of this process in 1872. And this is true, even if there had been no PVC molecule in the universe before Baumann.

Point (3) sounds baffling, especially in the light of the age-long dispute between rationalism and empiricism. We will return to this issue in the last section. At this stage, however, we are only concerned with the "physical representation of the formal system"; and, in this physical representation, $\Sigma \vdash \phi$ is no doubt a contingent fact of the physical world inside of the dotted line, the knowledge of which cannot be necessary, certain, or a priori. It can be known only by $a$ posteriori means, just like a chemical reaction. 
(II) What causes us to believe that, although it cannot be known a priori whether the computer will print out formula $\phi$ to the screen, still $\Sigma \vdash \phi$ "in the mathematical sense" is a necessary/a priori knowable truth? This belief comes from the following intuition: "The programmed computer is merely a physical representation of an abstract mathematical formal system in which $\Sigma \vdash \phi$ is true. If this physical representation is faithful then the computer necessarily prints out formula $\phi$ to the screen. If the computer happens not to print out $\phi$ to the screen, it only means that the physical representation is not faithful." But how can anybody know that $\Sigma \vdash \phi$ is true in the formal system "in the mathematical sense"? The usual idea is that it can be known to anyone who executes the formal derivation of $\phi$ from $\Sigma$ in the head. So what we actually do is represent the formal system in a brain and observe the behavior of the brain. Human brain is however not entirely reliable, so we prefer to execute the derivation in a brain+hand+pen+paper system. Anyway, from a physicalist point of view, it does not matter whether the formal system in question is represented in a human brain or a brain+hand+pen+paper system or any other physical system. As a matter of fact, we can represent the formal system in the mathematical sense in another computer; and if this second computer prints out $\phi$, we say that $\Sigma \vdash \phi$ is a priori true; and, accordingly, if the first computer also prints out $\phi$ to the screen, we say that it works properly; and the representation of the abstract/mathematical formal system in the first computer is faithful. But what would be the reason to put the second computer in such a privileged position? Upon what grounds can one physical representation be singled out as "the right one"? It is nonsense.

We have to recognize that the only sources of our mathematical knowledge are the formal systems embodied in concrete physical forms; and this knowledge can be obtained only by a posteriori means. From this point of view we must agree with the quantum computer theorists David Deutsch, Artur Ekert, and Rossella Lupacchini:

Numbers, sets, groups and algebras have an autonomous reality quite independent of what the laws of physics decree, and the properties of these mathematical structures can be just as objective as Plato believed they were (and as Roger Penrose now advocates). But they are revealed to us only through the physical world. It is only physical objects, such as computers or human brains, that ever give us glimpses of the abstract world of mathematics.

It seems that we have no choice but to recognize the dependence of our mathematical knowledge (though not, we stress, of mathematical truth itself) on physics, and that being so, it is time to abandon the classical view of computation as a purely logical notion independent of that of computation as a physical process. ([10], pp. 265-283) 
(III) In contrast to the first part of the above quoted passage, in my view, the ontological outcome of these epistemological considerations is the following: if there are no knowable truths other than the ones knowable from the physical world, there is no reason to be ontologically committed to the abstract/platonic things that these other truths would be about. But before drawing such a conclusion, let us consider a possible objection.

Many philosophers of mathematics, while admitting that a formal system is always represented in physical form, still assume that there is an "abstract structure" behind this physical representation, something that is represented. Sometimes we find the same ambivalent views in the formalist school. Curry writes:

[A]lthough a formal system may be represented in various ways, yet the theorems derived according to the specifications of the primitive frame remain true without regard to changes in representation. There is, therefore, a sense in which the primitive frame defines a formal system as a unique object of thought. This does not mean that there is a hypostatized entity called a formal system which exists independently of any representation. On the contrary, in order to think of a formal system at all we must think of it as represented somehow. But when we think of it as formal system we abstract from all properties peculiar to the representation. ([11], p. 30)

But, what does such an "abstraction" actually mean? What do we obtain if we abstract from some unimportant, peculiar properties of a physical system $L$ constituting a "physical representation of a formal system"? In order to think of this abstraction at all, in order to differentiate the important and unimportant features of physical system $L$, and to change from a more detailed representation of the system to a less detailed one, we must have a physical theory $(M, S)$ describing the physical system $L$ in question. That is to say, the whole abstraction - the result of the abstraction included - is described in $(M, S)$. However, the formal system $M$ also is "represented somehow", in Curry's terminology; it is another flesh and blood formal system. So, instead of obtaining a non-physical "abstract formal system" we remain to have flesh and blood formal systems.

Similarly, one cannot obtain an abstract formal system as an "equivalence class of isomorphic" flesh and blood formal systems $L_{1}, L_{2}, \ldots L_{n}$. To compare physical systems $L_{1}, L_{2}, \ldots L_{n}$ we have to use a physical theory $(M, S)$ which is capable of describing all $L_{1}, L_{2}, \ldots L_{n}$ together (Fig. 4). Only in a suitable $M$ it is meaningful to say that the theoretical representations of $L_{1}, L_{2}, \ldots L_{n}$ are isomorphic and constitute an equivalence class. Only in $M$ we can define the prototype of these structures, which can be regarded as an "abstract mathematical structure". But, all these objects live in the formal system $M$ which also is "represented somehow", that is, in a formal system existing in the physical world.

Thus, formal systems are always flesh and blood physical systems. These concrete physical systems should not be regarded as physical "representations" of some "abstract formal systems". Abstraction does not produce such abstract 


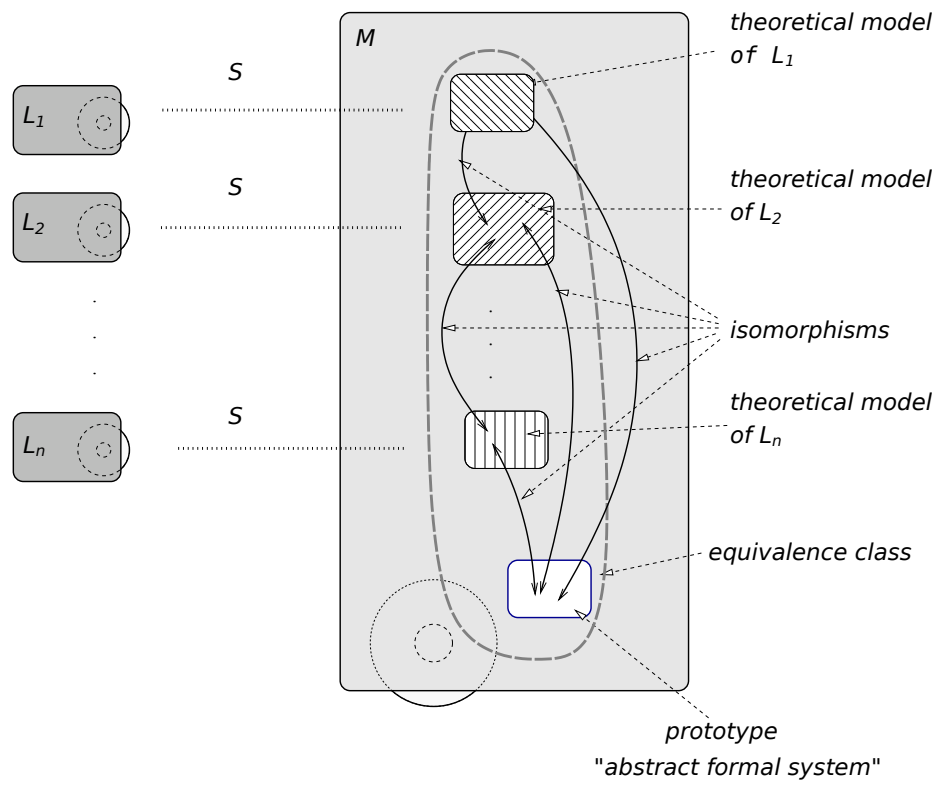

Figure 4: It is a categorical mistake to talk about "isomorphism" between physical systems. "Isomorphism" is a concept which is meaningful only in a formal system containing set theory. In order to say that "physical representations of formal systems" are "isomorphic" we need a physical theory $(M, S)$ in which the object systems are simultaneously represented

things over and above the physically existing formal systems - abstraction is a move from the concrete to the concrete.

Note that this claim does not deny the commitment to universals in the sense of scientific realism. When a satisfactorily confirmed physical theory $(L, S)$ claims that a physical object has a certain property adequately represented by means of a formal system $L$, then this reflects an objective feature of physical reality. When many different physical objects display a similar property that is describable by means of the same element of a formal system, then we may claim that these physical objects all possess the feature in question. This will be a true common feature of the group of objects in question - described by means of the elements of a formal system as a real physical system. This is also true for the description of some general features of a group of formal systems (as physical objects). But, this realist commitment does not entitle us to claim that "abstract structures" exist over and above the real formal systems of physical existence. Again, the reason is that if we tried to consider such an "abstract structure" as a feature of the formal system itself, or as a general feature of many similar formal systems, then we would only obtain some elements of another formal system of physical existence.

Finally, in view of what has been said, two consequences must be quite clear. 
First, the formal system $L$ used in a physical theory $(L, S)$ is itself a physical system. ${ }^{1}$ Second, a meta-mathematical theory describing a formal system $L_{1}$ is nothing but a physical theory $\left(L_{2}, S\right)$, where $L_{2}$ is a formal system (of physical existence) and the semantics $S$ points to the object formal system $L_{1}$ (as the physical system to be described).

To sum up, a formal system is a part of the physical world; the marks of the formal system are physical objects or physical phenomena related to the system and the derivation rules are embodied in those regularities that govern the system's behavior. The existence of a mathematical derivation, making a proposition of type $\Sigma \vdash \phi$ true, is a physical fact of the formal system as a part of the physical world. To prove a theorem is nothing but to observe this fact-for example, to observe a derivation process in a computer-that is, to observe a physical fact about a physical system. That is all.

Thus, physicalist account of mathematics completes the formalist foundation of mathematics, and removes the last residues of platonism, mentalism and immanent realism. ${ }^{2}$ The physicalist ontology of mathematical facts makes it completely pointless in mathematics to introduce a concept of "truth" different from that of being proved. A mathematical proposition, a theorem, as a formula in a formal system, does not carry meaning and semantic truth. At the same time, however, the fact that it is a theorem is a physical fact - a fact of the flesh and blood formal system itself. In this way, indeed, "mathematical truth is a part of objective reality" (Cf. [12]).

This is the way I propose to naturalize mathematics. In this way, mathematical knowledge is not conventional - there is nothing conventional in the statement $\Sigma \vdash X$. It is not trivial - sometimes it is highly non-trivial whether $\Sigma \vdash X$. It is not perfect, not a priori, and not certain. Just like non-mathematical sciences, mathematics delivers to us knowledge of contingent facts about a particular part of the physical world. Formal systems constitute this particular part of the physical world. This is what we can call "mathematical reality", and mathematicians rightly think themselves as scientists, exploring the intricacies of mathematical reality.

\footnotetext{
${ }^{1}$ Without entering into the details, a semantics $S$ also can be accounted for in physical terms.

${ }^{2}$ In order to make clear the distinction between my physicalist interpretation of mathematics and immanent realism, let me give one more example. ' $a^{2}+b^{2}=c^{2}$ ' is a theorem of Euclidean geometry, $\{$ Euclidean $\} \vdash a^{2}+b^{2}=c^{2}$. According to immanent realism, ' $a^{2}+b^{2}=c^{2}$, reflects some general truth about the real triangles in the physical world. Or at least, in the sense of the structuralist version of realism, the axiomatic theory "Euclidean geometry", as a whole, expresses some true structural property of the physical world; something about the congruence of rigid bodies, or the likes. Physicalism also claims that $\left\{\right.$ Euclidean $\vdash \vdash a^{2}+b^{2}=c^{2}$ expresses something in the physical world. But this something has nothing to do with the physical triangles and rigid bodies. \{Euclidean $\} \vdash a^{2}+b^{2}=c^{2}$ expresses a property of the formal system "Euclidean geometry", a property of the physical system consisting of the signs and the derivation mechanisms.
} 


\section{Induction versus deduction}

We have arrived at the conclusion that the knowledge we obtain through a deductive inference is nothing but an empirical knowledge we obtain through the observation of the formal system in question; through the observation of a physical fact of a physical system. Consequently, the certainty of mathematics, that is the degree of certainty with which one can know the result of a deductive inference, is the same as the degree of certainty of our knowledge about any other physical facts.

In order to explain the universal conviction that mathematical truths are necessary and certain, notice that there are many elements of our everyday knowledge which seem to be necessary and certain, but are actually obtained from inductive generalization. Break a long stick. We are "sure" about the outcome: the result is a shorter stick. This regularity of the physical world is known to us from experiences. The certainty of this knowledge is, however, no less than the certainty of the inference, say, from the Euclidean axioms to the height theorem.

Thus, mathematical and logical truths express objective facts of a particular part of the physical world, namely, the facts of the formal systems themselves. They are synthetic, a posteriori, not necessary, and not certain; they are fallible, but have contingent factual content, as any similar scientific assertion. On the other hand, the fact that the formal systems usually are simple physical systems of stable behavior and that the knowledge of mathematical and logical truths does not require observations of the world external to the formal systems explains, psychologically, why mathematical and logical truths appear to everyone to be necessary, certain and a priori.

The age-long rationalist-empiricist debate is based on the delusion that reasoning can deliver us truth of higher degree of certainty than inductive generalization. As we have seen, mathematical and logical truth is nothing but knowledge obtained through inductive generalization from experiences with respect to a particular physical system, the formal system itself. Since mathematical and logical derivation is reasoning par excellence, one must conclude that there is no higher degree of certainty than the one available in inductive generalization.

\section{Acknowledgments}

The research was partly supported by the OTKA Foundation, No. K100715.

\section{References}

[1] L. E. Szabó, Formal System as Physical Objects: A Physicalist Account of Mathematical Truth, International Studies in the Philosophy of Science $\mathbf{1 7}$ (2003) 117-125. 
[2] J. S. Mill, A System of Logic (Longmans, Green, and Company, London, 1843).

[3] D. M. Armstrong, A Theory of Universals (Cambridge University Press, Cambridge, 1978).

[4] P. Kitcher, The Nature of Mathematical Knowledge (Oxford University Press, Oxford, 1984).

[5] P. Maddy, Realism in Mathematics (Oxford University Press, Oxford, 1990).

[6] P. J. Davis and R. Hersh, The Mathematical Experience (Houghton Mifflin, Boston, 1981).

[7] J. Fang, Hilbert: Towards a Philosophy of Modern Mathematics, (Paideia Press, New York, 1970).

[8] D. Lewis, General semantics, in Semantics of Natural Language, eds. D. Davidson and G. Harman (D. Reidel Publishing Co., Dordrecht, 1972) I69228.

[9] M. Dummett, What Is Mathematics About?, in Mathematics and Mind, ed. A. George (Oxford University Press, Oxford, 1994) 11-26.

[10] D. Deutsch, A. Ekert, and R. Lupacchini, Machines: Logic and Quantum Physics, Bulletin of Symbolic Logic 6 (2000) 265-283.

[11] H. B. Curry, Outlines of a Formalist Philosophy of Mathematics (NorthHolland, Amsterdam, 1951).

[12] G. H. Hardy, Mathematical Proof, Mind 38 (1929) 1-25. 